\title{
ObTEnÇão de Nanopartículas de Cobre Para A Indústria Automotiva
}

\author{
Renato Souza e Silva ${ }^{1}$, Guilherme Wolf Lebrão ${ }^{2}$, Susana Marraccini Giampietri Lebrão ${ }^{3}$, \\ Viviane Tavares de Moraes ${ }^{4}$ \\ ${ }^{1}$ Instituto Mauá de Tecnologia \\ E-mails: silva.renato1997@gmail.com, guinet@maua.br, susana.lebrao@maua.br, \\ viviane.moraes@maua.br
}

\begin{abstract}
RESUMO
O avanço tecnológico e os fatores que estão ensejando a $4^{\mathrm{a}}$ Revolução Industrial têm impulsionado as pesquisas na área de reciclagem e de nanomateriais. $\mathrm{O}$ interesse na obtenção de nanopartículas de cobre veio pelas várias utilidades no meio automotivo e estrutural. Pesquisas mostram que a adição de nanocobre em óleos lubrificantes automotivos ajudam a amenizar o desgaste interno do motor, pois reduz o coeficiente de fricção entre o lubrificante e o interior do motor[1]. Além disso, o nanocobre é utilizado na matriz de alumínios[2] e de borrachas[3] para melhorar suas propriedades mecânicas, deixando-o mais resistente com um mínimo acréscimo em sua massa. Como objetivo deste trabalho tem-se a obtenção de nanopartícula de cobre a partir de resíduos de equipamentos eletroeletrônicos (REEE), proveniente de lixiviação por rota sustentável através de processos hidrometalúrgico[4]. A metodologia adotava envolve a síntese de nanopartículas de cobre, através de processo de redução química, onde adotou-se solução de sulfato de cobre purificado de processos hidrometalúrgicos e como agente redutor o ácido ascórbico. Espera-se como resultado definir uma rota de síntese de nanopartículas de cobre a partir de resíduos; por rota sustentável para obtenção de nanopartículas de metais para aplicação na indústria automotivas.
\end{abstract}

\section{INTRODUÇÃO}

Com o intenso avanço tecnológico e a constante inovação de equipamentos eletroeletrônicos por parte das grandes empresas, os aparelhos ficaram cada vez mais descartáveis e substituíveis pelos consumidores, o que gera uma enorme quantidade de resíduo de equipamento eletroeletrônico (REEE) ou sucata eletrônica[5], [6]. Esses resíduos muitas vezes podem conter metais tóxicos e outras substâncias que são prejudiciais ao meio ambiente se forem descartados de forma incorreta. Na indústria automobilística encontram-se cada vez mais sensores e equipamentos eletroeletrônicos como atrativos principalmente quando a sua utilização envolve melhor desempenho.

A reciclagem desses resíduos segue uma rota hidrometalúrgica sustentável, para a extração seletiva dos metais presentes[4], alguns com alto valor agregado (ouro, prata, platina e cobre)[7]. Para que os processos empregados nessa rota de reciclagem sejam viáveis e lucrativos, surgiu-se a necessidade de valorizar ainda mais os metais que foram separados, tratando-os e agregando valor com o refinamento de suas partículas. 
Com o aumento de pesquisas e o grande interesse na área da nanotecnologia, a síntese de nanopartículas de cobre, que é um dos metais mais encontrados nos REEEs[8], foi implantada afim de aumentar bruscamente seu valor no mercado, devido as diversas aplicações[9] na engenharia como um todo, mas mais especificamente na engenharia de materiais e automotiva.

O intuito da aplicação das nanopartículas de cobre nesse projeto é aprimorar a eficiência de equipamentos e dispositivos automotivos em geral, desde componentes para sustentação, impacto e segurança até para aditivo para óleos lubrificantes automotivos. As nanopartículas quando adicionadas em fluidos causam um aumento significativo da condutividade térmica quando comparado com fluidos convencionais[9], o que pode ser interessante em alguns casos no ramo automotivo.

\section{OBJETIVO}

Este artigo tem como objetivo definir uma rota de síntese de nanopartículas de cobre com resíduos de equipamentos eletroeletrônicos (REEEs) para aplicação automotiva. O trabalho também visa analisar as possíveis aplicações de nanopartículas de cobre na área da engenharia.

\section{SELEÇÃO DO RESÍDUO}

Os métodos de tratamento e separação dos resíduos foram desenvolvidos através da concentração de cobre obtida pela rota hidrometalúrgica de extração seletiva de placas de circuito impresso [4].

\section{MÉTODOS EXPERIMENTAIS}

\subsection{Materiais}

A preparação das nanopartículas foi feita em solução, utilizando sulfato de cobre pentahidratado $\left(\mathrm{CuSO}_{4} \times \mathrm{H}_{2} \mathrm{O}\right)$ com concentração de $1,96 \mathrm{~g} / \mathrm{L}$ e ácido ascórbico $\left(\mathrm{C}_{6} \mathrm{H}_{8} \mathrm{O}_{6}\right)$ em concentração de $40,8 \mathrm{~g} / \mathrm{L}$ e todos os ensaios foram realizado no IMT Instituto Mauá de Tecnologia.

\subsection{Preparação das nanopartículas de cobre}

A síntese da nanopartícula de cobre foi realizada pela reação de redução do sulfato de cobre pentahidratado e como agente redutor o ácido ascórbico[9], [10].

O experimento foi realizado com $100 \mathrm{~mL}$ de sulfato de cobre $(1,96 \mathrm{~g} / \mathrm{L})$ aquecida até próxima à temperatura de ebulição $\left(80^{\circ} \mathrm{C}\right)$, com agitação magnética sem que tivesse a ocorrência de vórtice. Após a estabilização da solução, foi gotejado $15 \mathrm{~mL}$ de ácido ascórbico $(40,8 \mathrm{~g} / \mathrm{L})$ na taxa de aproximadamente 1 gota por segundo. 


\section{RESULTADOS E DISCUSSÃO}

Os resultados obtidos a partir da redução química de cobre foram apresentados conforme mostra a Figura 1.

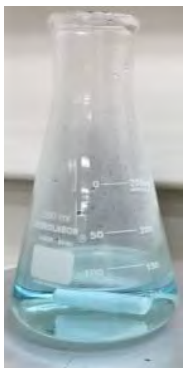

(A)

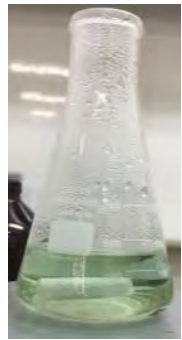

(B)

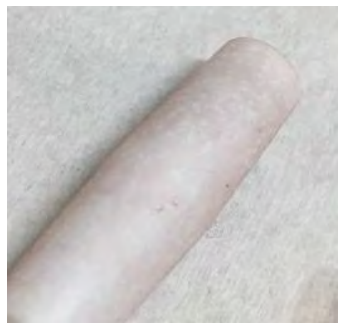

(C)

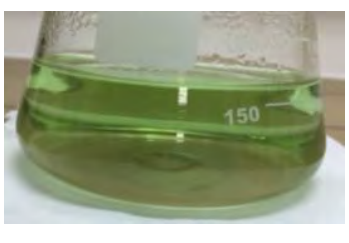

(D)

Figura 1 - Reação de redução de cobre em nanopartículas. (A) Solução precursora de nanopartículas, sulfato de cobre pentahidratado; (B) solução precursora após a adição do agente redutor, ácido ascórbico; (C) barra magnética com partículas aderidas, indicando a formação de nanopartículas de cobre; (D) Solução de cobre após $24 \mathrm{~h}$ da redução, com formação de precipitado alaranjado, de nanopartículas de cobre

O processo de redução de cobre ocorreu através de redução química, pode-se observar a formação de partículas na imagem da Figura 1 (C) e (D) com coloração característica de cobre em seu estado reduzido[9], [10].

A adesão de cobre metálico na superfície da barra magnética pode ocorrer devido a diferença de cargas na superfície dos materiais, pois o teflon que reveste a barra magnética possui cargas negativas enquanto que as nanopartículas de cobre possuem cargas positivas.

Após $24 \mathrm{~h}$, a solução final apresentou ainda mais características que evidenciaram a formação das nanopartículas, como o aumento da coloração verde e formação de um precipitado de cor característica do cobre.

\section{APLICAÇÕES DAS NANOPARTÍCULAS}

\subsection{Na engenharia de materiais}

$\mathrm{Na}$ área de materiais, o nanocobre tem ampla aplicação, podendo ser utilizado na matriz de borrachas, alumínios e nas superfícies de plástico[11]. Além de terem características magnéticas[12], no ensaio que foi realizado, foi evidenciado que as nanopartículas se agruparam ao redor da superfície do agitador magnético.

\subsubsection{Borracha de butadieno estireno (SBR)}

A borracha de butadieno estireno (SBR) é um elastómero muito difundido no mercado, no uso em diversas áreas, como: correias de transportadores, tubos, mangueiras e diversos artigos industriais. 
Com o incremento das nanopartículas de cobre na matriz da SBR conforme já foi feito por outros pesquisadores[3], teve êxito após verificada que o nanocobre ficam bem dispersas e distribuídas na matriz da borracha (SBR/NC). Essa adição implica em melhorias significativas nas propriedades da borracha.

Com relação à viscosidade, temos que a adição do nanocobre não altera o comportamento da sua pseudoplasticidade[13]-[15], porém influencia com um aumento na viscosidade da matriz quando submetido a baixas deformações.

As características a degradação térmica da SBR/NC não aumentam a resistência térmica, pelo contrário, há uma leve diminuição. Porém isso não ocorre quando há adição de nanocobre em polietileno de baixa densidade (NC/LDPE), onde as nanopartículas de cobre causam aumento estabilidade térmica nesses compostos plásticos[11].

A SBR pura tem propriedades elásticas conforme a literatura[16]. Com o incremento das nanopartículas de cobre houve um aumento de $81 \%$ na resistência a tração e de 55\% no módulo de elasticidade secante[3]. A melhoria nas características mecânicas se dá pela boa distribuição que as nanopartículas se dispõem na matriz da borracha [17].

\subsubsection{Alumínio}

A pesquisa feita com nanopartículas de óxido de cobre difundidas na matriz de alumínio [2], mostrou que a rugosidade superficial do alumínio reforçado com a nanopartícula teve um decréscimo de mais de $65 \%$ enquanto a porcentagem de massa de nanocobre foi aumentada de $1 \%$ para $2 \%$.

No artigo [2] também foi estudado que a dureza do material incrementado com as nanopartículas é aumentada conforme o aumento da quantidade de nanocobre adicionado, assim como acontece com a densidade, que sofre uma leve elevação, melhorando ainda mais suas propriedades mecânicas. A resistência a tração também é influenciada positivamente, sendo diretamente proporcional à porcentagem em massa da nanopartícula.

Outro estudo sobre as nanopartículas de cobre em alumínio [18] mostra que o nanocobre pode ser aderido a camada superficial do alumínio quando chocado a altas velocidades no substrato (Al).

\subsection{Na engenharia automotiva}

As aplicações vistas acima, na área de matérias, podem muitas vezes ser aplicadas na engenharia automotiva, na área de sustentação de componentes dos veículos, como as colunas, travessas, longarinas e assoalho, afim de melhorar suas propriedades mecânicas. No caso da borracha (SBR/NC), podem ser investidas em correias para transmissão em alto torque, visto que sua resistência mecânica foi melhorada. 
Porém há outras áreas em que a nanopartícula de cobre pode ser difundida.

\subsection{1. Óleo lubrificante automotivos}

Segundo estudo feito sobre a adição de nanopartículas de cobre à óleo do motor [1], o nanocobre diferentemente do cobre sólido traz benefícios quando se analisa o desgaste da superfície que o óleo entra em contato.

A explicação para tal melhoria no desgaste[19] vem de razões químicas, onde as substâncias presentes nos óleos de motores (anti-fricção e anti-desgaste) reagiriam com a parede da superfície de contato, com a adição das nanopartículas e à altas temperaturas, há uma reação de substituição que forma uma fina camada enriquecida pelo cobre.[1]

Foi comparado o coeficiente de fricção de óleos com e sem adição do nanocobre [20] e teve como resultado uma redução em média de $60 \%$ no coeficiente quando houve adição da nanopartícula ao óleo à $40^{\circ} \mathrm{C}$ e cerca de $30 \%$ em temperaturas mais elevadas.

\section{CONCLUSÃO}

A nanopartícula de cobre foi sintetizada através da reciclagem e separação por rota hidrometalúrgica sustentável do resíduo de equipamentos eletroeletrônicos (REEE), mais especificamente de placa mãe de circuito impressa moída (PCI-mãe-M). O nanocobre foi obtido por um ensaio simples e com um agente redutor amplamente conhecido e acessível, o que deve levar a um maior interesse na síntese e uso dessas nanopartículas no mercado futuramente.

Verificou-se que há diversas aplicações das nanopartículas de cobre em componentes e materiais que já são usados atualmente na indústria automotiva mundial e que o incremento das nanopartículas, podem apresentar melhorias substanciais em cada área de atuação do produto, visto que o nanocobre traz benefícios tanto em propriedades de materiais sólidos, como em fluidos lubrificantes de motores.

\section{REFERENCIAS}

[1] S. Tarasov, A. Kolubaev, S. Belyaev, M. Lerner, and F. Tepper, "Study of friction reduction by nanocopper additives to motor oil," Wear, vol. 252, no. 1-2, pp. 63-69, 2002.

[2] T. Rajmohan, K. Palanikumar, and S. Arumugam, "Synthesis and characterization of sintered hybrid aluminium matrix composites reinforced with nanocopper oxide particles and microsilicon carbide particles," Compos. Part B Eng., vol. 59, pp. 43-49, 2014.

[3] M. H. Harandi, F. Alimoradi, G. Rowshan, M. Faghihi, M. Keivani, and M. Abadyan, "Morphological and mechanical properties of styrene butadiene rubber/nano copper nanocomposites," Results Phys., vol. 7, pp. 338-344, 2017.

[4] M. P. K. Caldas, "Síntese de nanopartículas de prata a partir do reciclagem de placas de 
circuito impresso," p. 154, 2017.

[5] R. Hischier, P. Wäger, and J. Gauglhofer, "Does WEEE recycling make sense from an environmental perspective? The environmental impacts of the Swiss take-back and recycling systems for waste electrical and electronic equipment (WEEE)," Environ. Impact Assess. Rev., vol. 25, no. 5 SPEC. ISS., pp. 525-539, 2005.

[6] P. Dias, A. Machado, N. Huda, and A. M. Bernardes, "Waste electric and electronic equipment (WEEE) management: A study on the Brazilian recycling routes," J. Clean. Prod., vol. 174, pp. 7-16, 2018.

[7] J. Cui and L. Zhang, "Metallurgical recovery of metals from electronic waste: A review," J. Hazard. Mater., vol. 158, no. 2-3, pp. 228-256, 2008.

[8] A. C. Rodrigues, "Impactos Sócio-Ambientais Dos Resíduos De Equipamentos Elétricos E Eletrônicos : Estudo Da Cadeia Pós-Consumo No Brasil,” pp. 1-321, 2007.

[9] P. Gurav et al., "Stable colloidal copper nanoparticles for a nanofluid: Production and application," Colloids Surfaces A Physicochem. Eng. Asp., vol. 441, pp. 589-597, 2014.

[10] A. Guzman, J. Arroyo, L. Verde, and J. Rengifo, "Synthesis and Characterization of Copper Nanoparticles/Polyvinyl Chloride (Cu NPs/PVC) Nanocomposites," Procedia Mater. Sci., vol. 9, pp. 298-304, 2015.

[11] X. Xia, S. Cai, and C. Xie, "Preparation, structure and thermal stability of Cu/LDPE nanocomposites," Mater. Chem. Phys., vol. 95, no. 1, pp. 122-129, 2006.

[12] A. Reznickova, M. Orendac, Z. Kolska, E. Cizmar, M. Dendisova, and V. Svorcik, "Copper nanoparticles functionalized PE: Preparation, characterization and magnetic properties," Appl. Surf. Sci., vol. 390, pp. 728-734, 2016.

[13] S. Sadhu and A. K. Bhowmick, "Unique rheological behavior of rubber based nanocomposites," J. Polym. Sci. Part B Polym. Phys., vol. 43, no. 14, pp. 1854-1864, 2005.

[14] S. K. Lim, J. W. Kim, I. J. Chin, and H. J. Choi, "Rheological properties of a new rubbery nanocomposite: Polyepichlorohydrin/organoclay nanocomposites," J. Appl. Polym. Sci., vol. 86, no. 14, pp. 3735-3739, 2002.

[15] Y. Zhong and D. De Kee, "Morphology and properties of layered silicate-polyethylene nanocomposite blown films," Polym. Eng. Sci., vol. 45, no. 4, pp. 469-477, 2005.

[16] S. Sadhu and A. K. Bhowmick, "Preparation and properties of styrene-butadiene rubber based nanocomposites: The influence of the structural and processing parameters," J. Appl. Polym. Sci., vol. 92, no. 2, pp. 698-709, 2004.

[17] N. M. Barkoula, B. Alcock, N. O. Cabrera, and T. Peijs, "Fatigue properties of highly oriented polypropylene tapes and all-polypropylene composites," Polym. Polym. Compos., vol. 16, no. 2, pp. 101-113, 2008.

[18] V. V. Pogorelko, A. E. Mayer, and V. S. Krasnikov, "High-speed collision of copper nanoparticle with aluminum surface: Molecular dynamics simulation," Appl. Surf. Sci., vol. 390, pp. 289-302, 2016.

[19] W. Dai, B. Kheireddin, H. Gao, and H. Liang, "Roles of nanoparticles in oil lubrication," Tribol. Int., vol. 102, pp. 88-98, 2016.

[20] F. L. Guzman Borda, S. J. Ribeiro de Oliveira, L. M. Seabra Monteiro Lazaro, and A. J. Kalab Leiróz, "Experimental investigation of the tribological behavior of lubricants with additive containing copper nanoparticles," Tribol. Int., vol. 117, pp. 52-58, 2018. 